\title{
Desnutrición en hijos de madres adolescentes
}

\author{
Dra. Myrna Jopia R.; Dra. Yolanda Muñoz L.'
}

Undernutrition in infants born to adolescent mothers

The frecuency of undernutrition in infants born to mothers aged 17 years or less (study) was compared to that of first infants born to 18 to 35 year old women (controls) of the same low socioeconomic condition, followed in an outpatient clinic at metropolitan Santiago, Chile. Undernutrition occurred in $17.7 \%$ of children born to adolescent females vs only $5.7 \%$ in controls. There were no premature infants in the study group and only one such patient was included among controls. No differences w'ere detected in birth weight, level of attained education and frecuency of married mothers, but only $11.4 \%$ of the study group were economically dependent from the father in contrast with $73.5 \%$ of cases among control patients.

(Key words: infants from adolescent mothers, frecuency of undernutrition).

El embarazo en la adolescente involucra para el adolescente y su familia un problema de tipo socjoeconómico, cultural biológico y psicológico.

Las embarazadas que atiende el consultorio Los Nogales provienen de un medio de condición socioeconómica baja con todas las características que ello implica en cuanto a su forma y calidad de vida.

Partiendo del conocimiento de que el embarazo precoz, llamese asi al embarazo en adolescen. tes menores de 17 años, es un factor de riesgo en la incidencia y prevalencia de la desnutrición infantil, quisimos cuantificar dicha relación en este nivel de atención primaria, con el objeto de verificar si se cumple esa regla, y los factores concurrentes.

\section{PACIENTES Y METODO}

Dutante el año 1984, 52 adolescentes menores de 17 años controlaron su embarazo en el consultorio Los Nogales, ubicado en el área œentral de Santiago con una población asignada de 28.178 habitantes. Se descartaron 17 pacientes por abortos, mortinatos, canbios de residencias y falsos domicilios, de modo que ingresaron al estudio 35 adolescentes de hasta 17 años de edad (promedio 16,2 años) que finalizaron su embarazo.

1 Médicos Integrales Servicio Salud Central, Area Metropolitana.
EI grupo control quedó formado por 35 embarazadas de 18 a 35 años de edad (promedio cs de 20,8 años) primigestas, elegidas al azar.

La información necesaria fue obtenida a través del análisis de la ficha clínica de control maternal; la ficha clínica de los lactantes y una encuesta domiciliaria.

Las evaluaciones nutricionales se realizaron mediante tablas peso-talla, y de incremento ponderal mensual, utilizadas habitualmente en los consultorios del Servicio Nacional de Salud, para el primer año de vida de los niños. La información necesaria fue obtenida del contro] de salud más próximo a la ejecución del estudio en cada caso, fluctuando las edades entre 4 y 12 meses para ambos grupos, promedio 8,8 meses en los niños controles y 9,1 meses en los hijos de adolescentes.

\section{RESULTADOS}

Entre los hijos de adolescentes de detectó una frecuencia de $17,1 \%$ de desnutrición, en cambio en el grupo control sólo ocurrió en $5,7 \%$, frecuencia tres veces menor que en el grupo en estudio (tabla 1).

El peso de nacimiento en $68,5 \%$ de los niños de madres adolescentes fue mayor de 3 kilos, 10 mismo ocurrió en $65,7 \%$ en el grupo control. Los pesos insuficientes $(2,3$ a $3 \mathrm{~kg})$ ocurrieron con frecuencias de 28,5\% en ambos grupos; el bajo peso $(<2,5 \mathrm{~kg})$ en $2,8 \%$ en el primer grupo $y$ $5,6 \%$ en el grupo de control. No hubo casos de prematuridad en el grupo de madres adoles. 
Tabla 1.

Estado nutritivo del niño

\begin{tabular}{lrrrrr}
\hline & \multicolumn{2}{c}{ Grupo I } & & \multicolumn{2}{c}{ Grupo II } \\
\cline { 2 - 3 } \cline { 5 - 6 } & $n$ & $\%$ & & $n$ & $\%$ \\
\hline Normal & 27 & 77.1 & 31 & 88,5 \\
Desnutrido & 6 & 17,1 & 2 & 5,7 \\
Sobrepeso & 2 & 5.7 & & - & - \\
Alto riesgo & - & - & & 5,7 \\
\hline \hline Total & 35 & 100 & 35 & 100
\end{tabular}

Tabla 2.

Peso del recitun nacido

\begin{tabular}{lrrrrr}
\hline & \multicolumn{2}{c}{ Grupol } & & \multicolumn{2}{c}{ Grupo II } \\
\cline { 2 - 3 } \cline { 5 - 6 } & \multicolumn{1}{c}{$\mathrm{n}$} & $\%$ & & \multicolumn{1}{c}{$\mathrm{n}$} & $\%$ \\
\hline$+3 \mathrm{~kg}$ & 24 & 68,5 & & 23 & 65,7 \\
$2,5-3 \mathrm{~kg}$ & 10 & 28,5 & & 10 & 28,5 \\
$-2,5 \mathrm{~kg}$ & 1 & 2,8 & & 5.6 \\
\hline Total & 35 & 100 & 35 & 100
\end{tabular}

Tabla 3.

F.scolaridad madres

\begin{tabular}{lccccr}
\hline & \multicolumn{2}{c}{ Grupo I } & & \multicolumn{2}{c}{ Grupo II } \\
\cline { 2 - 3 } \cline { 5 - 6 } & $n$ & $\%$ & & $n$ & $\%$ \\
\hline Básica & 25 & 71,4 & & 11 & 35,4 \\
Media & 10 & 28,5 & & 20 & 57,5 \\
I!niversitaria & - & - & & 2 & 5,7 \\
\hline \hline Total & 35 & 100 & 35 & 100
\end{tabular}

centes, y sólo uno en el grupo de control (tabla 2).

No se encontraron diferencias en la frecuencia y tipo de morbilidad entre ambos grupos.

Las evaluaciones del ambiente socio-cultural se efectuaron en base a la encuesta. Nos referimos a los tres aspectos sociales que nos parecen interesantes de presentar por sus caracterj́sticas.

Uno de ellos fue la escolaridad de las madres: entre las madres adolescentes, sólo $28,5 \%$ habian cursado algún nivel de enseñanza media, el resto $(71,4 \%$ llegó sólo a la enseñanza básica). En el grupo control, en cambio $57.1 \%$ l legó a la enseñanza media e incluso $5,7 \%$ tenía algún grado de instrucción superior (tabla 3).

En conjunto, sólo $40 \%$ de las parejas del grupo en estudio $y$ 48,5\% del grupo control tenia enseñanza media. En ambos grupos las madres desconocían la escolaridad de la pareja en $31,4 \%$ y $28,5 \%$ respectivamente.

En cuanto al estado civil, $40 \%$ de las adolescentes declararon estar casadas antes del parto $y$ sólo $51,5 \%$ después de él. En el grupo control las casadas después del parto aumentaron de 48,5\% a $62,8 \%$ (tabla 4).

Los hjjos de adolescentes sólo en $11,4 \%$ de. pendian económicamente de su progenitor, y cn $57,1 \%$ de sus abuelos, en cambio los niños del grupo control dependian en 73,5 de los casos del progenitor.

\section{COMENTARIO}

Durante la consulta diaria nos habiamos dado cuenta que los hijos de madres adolescentes tenian tnayores dificultades en el desarrollo psicomotor y nutricional. Quisimos verificar esta observación e impresión realizando este cstudio ${ }^{1}$.

Tabla 4.

Estado cívi]

\begin{tabular}{|c|c|c|c|c|c|c|c|c|}
\hline & \multicolumn{4}{|c|}{ Grupo 1} & \multicolumn{4}{|c|}{ Grupo 11} \\
\hline & \multicolumn{2}{|c|}{ Antes parto } & \multicolumn{2}{|c|}{$\overline{\text { Después parto }}$} & \multicolumn{2}{|c|}{ Antes parto } & \multicolumn{2}{|c|}{ Después parto } \\
\hline & $\pi$ & $\%$ & $\pi$ & $\%$ & $\mathrm{n}$ & $\%$ & $\bar{n}$ & $\%$ \\
\hline Casadas & 14 & 40,0 & 18 & 51,5 & 17 & 48,5 & 22 & 62,8 \\
\hline Solteras & 17 & 48,5 & 15 & 428 & 12 & 36.3 & 11 & 35,4 \\
\hline Conviven & 4 & 11,4 & 2 & 5,7 & 2 & 6,0 & 2 & 5,7 \\
\hline Ignorado & - & - & - & - & 2 & 5,7 & - & - \\
\hline Total & 35 & 100 & 35 & 100 & 35 & 100 & 35 & 100 \\
\hline
\end{tabular}


Además nos llamaba la atención la actitud con. formista de estas madres y la indiferencia del medio frente a los problemas del embarazo pre$\mathrm{coz}$, pudiendo constatar, en nuestra revisión bibliográfica, que el hecho era aceptado como común y habitual dentro de su nivel social. ${ }^{2}$

Es conocido que la edad materna es un factor de riesgo para el feto: en las madres muy jóvenes se concentran con mayor frecuencia la insuficiencia placentaria con la importante consecuencia del bajo peso al nacer y el parto prematuro ${ }^{3}$. Posteriormente estos niños estarían más expues. tos al riesgo de enfermar y de sufrir deficiencias nutricionales durante la lactancia ${ }^{4}$. Pensábamos pues que la desnutrición de los hijos de adolescentes estaría influenciada por estos factores. Sin enibargo, los resultados obtenidos en este trabajo descartaron estas hipótesis, ya que los nir̃os que sufrieron desnutrición no tenf́atl pesos bajos al nacer ni prematurez en comparación con otros reportes ${ }^{5,6,7}$. Tampoco encontramos diferencias de morbilidad que pudiesen repercutir sobre el desarrollo ponderal.

A pesar de ello, los hijos de madres adolescentes presentaron tres veces mayor frecucncia de desnutrición que en el grupo control. ¿A qué se debe esta tendencia? Es indudable que el medio tiene para estos niños una serie de desventajas: la interrupción de la educación de los padres es una consecuencia inherente al embarazo precoz ${ }^{7,8}$, ella estaría limitando sus conocimientos y preparación, reduciendo su destreza para educar y criar a los hijos y sus posibilidades de enfrentar positivamente los desafios $y$ adversidades del medio ${ }^{9}$. Este $y$ otros trabajos demues. tran el alto porcentaje de soltería en la adolescente embarazada $1,2,5,7,10,11$. La falta de legalización del matrimonio, restaría solidez al grupo familiar; lógicamente es probable que debido a la edad de las madres no pueda aún esperarse un nivel de madurez que dé sentido de responsabilidad a esta unión de pareja, que suele comenzar siendo puramente accidental, sin preparación previa o deseo real de formar una familia. Desde ahí en adelante el desarrollo de estos niños puede resultar de un conjunto de situaciones obligadas por circunstancias dejadas al azar. Aunque tampocó encontramos cifras que demostraran que la situación es categóricamente mejor en el grupo control, debido a características propias de esta población en su comportamiento socio-cultural $^{2}$ el f́enómeno es más marcado y posible- mente más perjudicial en la adolescencia. Por último la dependencia económica no centrada en los padres disgrega $o$ anula aún más la responsabilidad de éstos y limita su participación como actores principales en la formación de su propio grupo familiar, perdiéndose el valor de éste ${ }^{2}$.

Los logros alcanzados en el último tiempo en salud materno infantil, han sido indudablemente muy importantes en ntrestro pais; sin embargo el problema de la desnutrición infantil continua siendo un inquietante desafío. E1 medio ambiente puede ser uno de los principales factores en estos fenómenos, por ello parece perentorio aumentar la participación de otros sectores e instituciones para enfrentar estos problemas me. diante un trabajo integral $y$ organizado para fomentar cambios de conductas favorables a las personas y las familias.

\section{RESUMEN}

Este trabajo tiene como objeto verificar la relación que existe entre embarazo precoz y desnutrición que existe entre embarazo precoz y 35 madres menores de 17 años que se controlaron su embarazo en el consultorio Los Nogales durante el año 1984. Se encontró un porcentaje de $17,1 \%$ de niños desnutridos en estas madres; cifras tres veces superior a la encontrada en un grupo control constituido por madres primigestas entre 18 y 35 años controladas durante igual período de tiempo en el mismo consultorio. Se descartaron las posibles etiologías de bajo peso de nacimiento, prematurez, ya que los niños desnutridos de nuestro grupo en estudio tuvieron un peso de nacimiento superior a tres kilos, y tampoco se encontraron patologías que deterioraran el estado nutricional durante el primer año de vida. Sin embargo es interesante destacar los factores sociales desfavorables del medio y ellos son: la interrupción de la escolaridad ya que sólo un $28,5 \%$ de las adolescentes lcgra cursar la enseñanza media; sólo un $51,5 \%$ forma una convivencia estable legalizando su matrimonio; y finalmente sólo en un $11,4 \%$ estos niños depen. den económicamente de sus progenitores. Puede concluirse que la desnutrición observada en los hijos de madres adolescentes corresponde a desnutrición relacionada con los factores socjo-culturales y económicos. 


\section{AGRADECIMIENTOS}

A María Isabel Soto y Vicenza Casanueva, Matronas del consultorio Los Nogales por su valiosa cooperación.

\section{REFERENCIAS}

1. Wels W., Cuadro L., Papic Z., Asludello $C_{\text {. }}$ Condiciones socioeconómicas, culturales familiares de una población de gestantes precoces controladas en un consultorio de Santiago. XVIII Congreso Chileno de Obstetricia y Ginecología; 1980

2. Largo E.: Madres solteras adolescentes de estado sociocconómíco bajo; Tesis para optar al grado de licenciado en Antropología 1979; 69.70.

3. Weneghello I.: Pediatría, $3^{\text {ta }}$ Edición. Editorial Mediterráneo, Santiago Chile, 1985: 184-192.

4. Meneghello 1.: Pediatría $3^{\text {ral }}$ edición. Editorial Modjterráneo, Santiago Chile 1985; 116-121.

5. Sismondi P.. Volonse R. Grae M.: lil embarazo y parto en la adolescentc; Rev Chil Obstr Gin 1984; 49: $41-45$.
6. Rubio R., Fuentes A., Sanhueza E., Rodriguez G., Ortiz M.: Reproducción en la adolescencia. Rev Chil Obstetr Gin 1981: 46, 112-115.

7. Martinez E., Weld E., Iglesias J. GomezR.; Brito $C$. Olguin $M$. Vásquez $M$. .: Experiencia en una Lnidad de gestantes prococes; Rev Chil Obstetr Gin 1985; $50,127-139$.

8. González E., Orellane M., Alarcón G., Romero $M$. Molina R., Garcia $L$, Jentzen G., Parada $E$.: Características de adolescentes embarazadas en situación irregular, XX Congreso Chileno de Obstetricia, Ginecología, 1983.

9. Monkeberg $F_{1}$ : Jaque cl subdesarrollo. Parte III, No 2, 4a. Ed. Santiago-Chile. Fditorial Gabriela Mistral, 1976: 132-135.

10. Hommel P., Asen D., Andrade M., Adolescentes embarazadas de sectores populares urbanos; características sociodemográficas y familiares. Rer Chil de Obstetr Gin 1981;4: 163-172.

11. Matus $C_{\text {. }}$ Leiva $H$, Beltrón $R$., Jorquera $M$., Valdeira M., Peretra B., Schmol A.: XVIlI Congreso de Obstetricia y Ginecologia, 1980. Condición sacial social de la madre adolescente en srica. 